\title{
An Open-label Randomized Clinical Study to Compare the Effects of a Nutritional Supplement versus Vitamin E on Fibroscan Score in Nonalcoholic Steatohepatitis (NASH) Patients
}

\author{
Shahed Ashraf ${ }^{1, *}$, Jamshed Alam¹, Jahangir Alam Sarkar ${ }^{1}$, Faiz Ahmed Khondaker ${ }^{1}$, \\ Yusuf Farhana ${ }^{2}$, Naila Atik Khan ${ }^{3}$ \\ ${ }^{1}$ Department of Hepatology, Shaheed Suhrawardy Medical College Hospital, Sher- E- Bangla Nagar, Bangladesh \\ ${ }^{2}$ Department of Pathology, Bangabandhu Sheikh Mujib Medical University, Dhaka, Bangladesh \\ ${ }^{3}$ Department of Biochemistry \& Molecular Biology, Bangabandhu Sheikh Mujib Medical University, Dhaka, Bangladesh
}

Copyright $\bigcirc 2018$ by authors, all rights reserved. Authors agree that this article remains permanently open access under the terms of the Creative Commons Attribution License 4.0 International License

\begin{abstract}
Background: Despite the benefit of lifestyle changes, there is no standard treatment for Fibrotic NASH. However the concept of reversibility of liver fibrosis and cirrhosis with various natural biologically active compounds and antioxidant micro-nutrients is not new. Aim: The aim of this study was to compare effectiveness of Viusid $^{\circledR}$ (a nutritional supplement) and Vitamin $E$ in reducing steatosis and liver fibrosis score in patients with fibrotic NASH. Methods: 52 patients diagnosed with nondiabetic and noncirrhotic NASH on liver fibroscan were divided into 2 groups randomly and given Viusid ${ }^{\circledR} 3$ sachets daily to 25 patients or Vitamin E 800 IU daily to 27 patients along with a hypocaloric diet and exercise for 3 months. Results: After 3 months treatment with Viusid ${ }^{\circledR}$, as compared with Vitamin E, was associated with a significant reduction of both mean steatosis score (CAP value reduces from $286 \pm 16.3$ to $208 \pm 18.5 \mathrm{~dB} / \mathrm{m}$ in Viusid $^{\circledR}$ group vs. from $278 \pm 14.4$ to $253 \pm 12.1 \mathrm{~dB} / \mathrm{m}$ in Vitamin $\mathrm{E}$ group; $\mathrm{p}<0.00001)$ and fibrosis score $(E$ value reduces from $6.8 \pm 0.5$ to $5.1 \pm 0.7 \mathrm{kPa}$ in $\mathrm{Viusid}^{\circledR}$ group vs. from $6.9 \pm 0.5$ to $6.5 \pm 0.4 \mathrm{kPa}$ in Vitamin $\mathrm{E}$ group; $\mathrm{p}<0.00001$ ). Similarly, the mean alanine transaminase (ALT) levels also significantly decreased from $114 \pm 25.9 \mathrm{U} / \mathrm{L}$ to $43 \pm 9.1 \mathrm{U} / \mathrm{L}$ in Viusid ${ }^{\circledR}$ group compared to from $105 \pm 15.5 \mathrm{U} / \mathrm{L}$ to $55 \pm 11.7 \mathrm{U} / \mathrm{L}$ in vitamin $\mathrm{E}$ group $(\mathrm{p}>0.00001)$. Conclusions: Viusid $^{\circledR}$ was superior to Vitamin $\mathrm{E}$ in reducing steatosis \& fibrosis score in nondiabetic and noncirrhotic NASH patients. However, further large scale trial is needed to better assess the value of Viusid $^{\circledR}$ for fibrotic NASH management.
\end{abstract}

Keywords Steatosis, Nonalcoholic Steatohepatitis, Fibrosis, Antioxidant Micro-nutrients, Viusid ${ }^{\circledR}$, Vitamin E

\section{Introduction}

Nonalcoholic fatty liver disease (NAFLD) is defined as excessive fat (i.e., triglyceride) accumulation in the liver without secondary hepatic fat accumulation such as significant alcohol consumption, use of steatogenic medication, or hereditary disorders [1]. It is histologically categorized into nonalcoholic fatty liver (NAFL) and nonalcoholic steatohepatitis (NASH). NAFL is defined as the presence of hepatic steatosis without hepatocellular injury, whereas NASH is characterized by NAFL with hepatocellular ballooning injury with or without fibrosis [1]. NAFLD is now the most common cause of chronic liver disease worldwide [2] and affects $15 \%$ to $30 \%$ of the population but is more prevalent (about 50\%-90\%) in patients with diabetes, metabolic syndrome, and severe obesity[1,3]. Recently, the prevalence of NAFLD in general population of Bangladesh has been estimated to vary from 4 to $18.4 \%$ and it is increasing day by day [4]. About $40 \%$ of them are nonalcoholic steatohepatitis (NASH). They are in danger of progression to cirrhosis and hepatocellular carcinoma (15-19\%) in next 5 to 10 years [4]. Now NAFLD is becoming a major concern among other liver diseases and management of NASH with progressive fibrosis has become a big challenge for hepatologists. Sedentary lifestyle, modern western nutrition, and genetic predispositions have been identified as major causes of NAFLD. These lead to liver injury via insulin resistance and an excess of free fatty acids in hepatocytes, resulting in oxidative stress and lipotoxicity (lipid-peroxidation) that promote the activation of intracellular stress kinases and apoptosis or necroapoptosis (NASH) [5]. The damaged hepatocytes directly trigger 
inflammation and fibrogenesis, but can also lead to the emergence of fibrogenic progenitor cells. Moreover, NASH is linked to inflammation in peripheral adipose tissues that involves mainly macrophages and humoral factors, such as adipokines and cytokines $[4,5]$. Therefore, the treatment strategies for NAFLD aim to improve insulin sensitivity, modify underlying metabolic risk factors or to protect the liver from further insult by reducing oxidative stress and inflammatory markers [6]. So far, there is no established pharmacological treatment for NAFLD. The most efficient treatment is lifestyle modification by diet, exercise and weight loss as the mainstay of therapy $[1,6]$, but (adjunctive) pharmacological strategies are urgently needed. Multiple pharmacological interventions have been attempted with variable success. These include pentoxifylline, orlistat, Vitamin E, ursodeoxycholic acid, omega - 3 fatty acids, and insulin sensitizers and lipid-lowering agents [6]. However, the American Association for the Study of Liver Diseases (AASLD) recommends the use of Vitamin $\mathrm{E}$ administered at daily doses of $800 \mathrm{IU} /$ day in non-diabetic adults with biopsy-proven NASH and is considered as a first line pharmacotherapy for this patient population [7].

As lipid peroxidation (malondialdehyde), overabundance of reactive oxygen species (ROS) and pro-inflammatory cytokines (IL-2, IL-6 and TNF-alpha) are key mediators in the progression from relatively stable hepatic steatosis to potentially progressive steatohepatitis, thus, there is a rationale of using potential antioxidant, anti-inflammatory and immunomodulator based micro-nutrients to dietary measures and exercise to halt the progression of the disease [8]. Viusid ${ }^{\mathbb{B}}$ (Catalysis Laboratory, Spain), a nutritional supplement, has different molecules (Glycyrrhizic acid, Glucosamine, Ascorbic acid, Zinc and other micro-nutrients) with recognized antioxidant, immunomodulatory and anti-fibrotic properties (Table 1) [8,9]. Antioxidant activity of glycyrrhizic acid, the most important active ingredient of the supplement, was found to be over 100 times stronger than that of Vitamin E [10]. Furthermore, the manufacturer used a patented bio-technology namely "Molecular Activation" to activate and increase the overall antioxidant activity of Viusid $^{\mathbb{B}}$ (before activation the antioxidant capacity was 1.1 points and after activation the antioxidant capacity significantly increased to 9.9 points) which is about $800 \%$ more potent than its previous non-activated state (Figure 1) $[9,11,12]$. Evidences showed that Viusid ${ }^{\mathbb{B}}$ inhibits oxidative stress, lipid per-oxidation (MDA) and several pro-inflammatory cytokines (TNF-alpha, IL-1 \&IL-6) involved in fibrogenesis process. Moreover it demonstrated different anti-inflammatory effects (increased production of IL-10), anti- apoptotic effect of healthy hepatocyte, apoptotic stimulating effect of hepatic stellate cells (HSC), hepatocyte proliferating effect and stabilizing effect of hepatic cellular membranes which are potential for "Anti-fibrotic Activity" [13, 14].
Table 1. Composition of Viusid ${ }^{\circledR}$

\begin{tabular}{|l|c|}
\hline \multicolumn{1}{|c|}{ Ingredients } & $\begin{array}{c}\text { Average Values } \\
\text { (per 4 g sachet) }\end{array}$ \\
\hline Glycyrrhizic acid & $0.033 \mathrm{~g}$ \\
\hline Ascorbic acid & $0.020 \mathrm{~g}$ \\
\hline Folic acid & $66.00 \mu \mathrm{g}$ \\
\hline Zinc sulfate & $0.005 \mathrm{~g}$ \\
\hline Glucosamine & $0.666 \mathrm{~g}$ \\
\hline Arginine & $0.666 \mathrm{~g}$ \\
\hline Glycine & $0.333 \mathrm{~g}$ \\
\hline Malic acid & $0.666 \mathrm{~g}$ \\
\hline Pyridoxal & $0.60 \mathrm{mg}$ \\
\hline Cyanocobalamine & $0.30 \mu \mathrm{g}$ \\
\hline Calcium pantothenate & $0.002 \mathrm{~g}$ \\
\hline
\end{tabular}

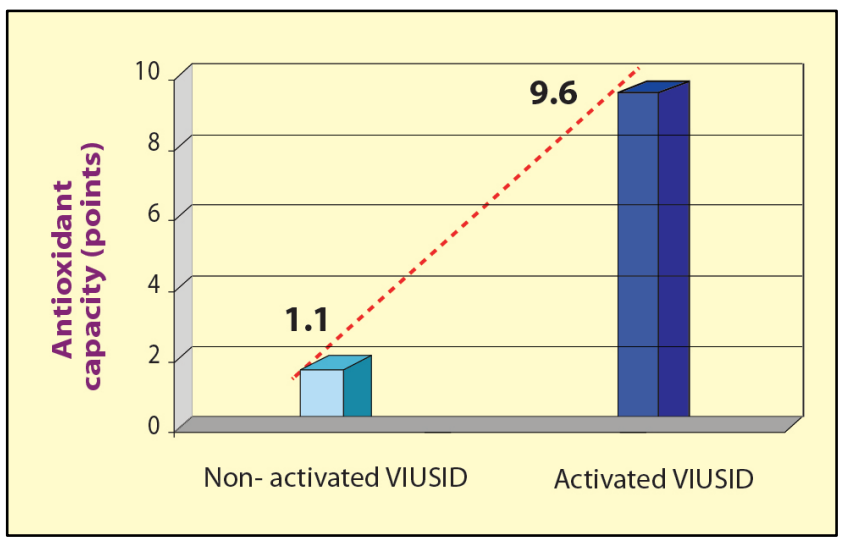

Figure 1. Impact of Molecular Activation Technology on the antioxidant capacity of Viusid ${ }^{\circledR}$

Encouraging effects of Viusid ${ }^{\circledR}$ on liver histology and biochemistry have been reported in patients with non-alcoholic fatty liver disease and chronic hepatitis C. The authors reported that the addition of Viusid ${ }^{\circledR}$ to the conventional interferon/ribavirin therapy was associated with significant histological and biochemical improvements, especially in patients without a sustained virological response [15]. In another study, the same authors showed that the administration of Viusid ${ }^{\circledR}$ combined with a lifestyle modification based on a hypocaloric diet and exercise during 6 months was associated with marked histological improvements in steatosis, lobular inflammation, ballooning and NAFLD activity score in patients with non-alcoholic fatty liver disease [8]. Additionally, another study demonstrated a significant biochemical improvement of $\mathrm{Viusid}^{\circledR}$ in achieving ALT normalization in NAFLD patients [9]. No significant clinical and laboratory adverse events (AEs) have been reported with the use of Viusid ${ }^{\circledR}$ in previous trials $[8,9,13,14,15]$.

Thus, the aim of this study was to compare the effectiveness of Viusid $^{\circledR}$ and Vitamin $E$ in reducing steatosis and liver fibrosis, administered for three months in patients with fibrotic NASH. 


\section{Materials and Methods}

Patients: This is an open-label randomized clinical study carried out at the Hepatology Department of Shaheed Suhrawardy Medical College Hospital in Dhaka, Bangladesh from June 2015 to September 2016. The study included 52 patients diagnosed with non-cirrhotic NASH on fibroscan of liver, with raised serum ALT level. Trial profile of the patients is shown in Figure 2. Some characteristics and parameters of participants are shown in Table 2. All participants provided written informed consent before enrolment and all responses were kept confidential.

Inclusion criteria included male and female non-diabetic NAFLD patients of 28-62 years of age, diagnosed with non-cirrhotic NASH on fibroscan of liver, with raised serum ALT level, absence of significant alcohol consumption (weekly ethanol consumption of $<20 \mathrm{~g}$ ) and ability to provide informed consent.

Exclusion criteria included patients having history of alcohol consumption, diabetes, presence of any other form of liver disease, positive screening for viral hepatitis B and $\mathrm{C}$, pregnancy or lactation, decompensated cirrhosis, use of drugs such as ursodeoxycholic acid, betaine, pioglitazone, rosiglitazone, metformin, pentoxifylline or gemfibrozil, use of cholesterol-lowering statin drugs, severe or morbid obesity (body mass index $\pm 35 \mathrm{~kg} / \mathrm{m}^{2}$ ), refusal to participate in the study, and those with other hepatic diseases including autoimmune hepatitis, wilson's disease, hemochromatosis and alpha-1 antitrypsin deficiency, concomitant disease with reduced life expectancy, severe psychiatric conditions and drug dependence. Patients with hepatic steatosis only were not recruited for the trial.

Study design: All patients were divided into two groups randomly and administered Viusid ${ }^{\mathbb{B}} 3$ oral sachets (each containing $4 \mathrm{~g}$ powder) daily to 25 patients (Group I) or Vitamin E at a dose of 800 IU daily to 27 patients (Group II) along with a lifestyle modification program consisting of hypocaloric diet $(1500 \pm 100 \mathrm{kcal} ; \quad 50-60 \%$ of carbohydrates, $20-25 \%$ of lipids and $15-20 \%$ of proteins) and exercise (walking / jogging; $40 \mathrm{~min} /$ day at least 5 days a week) for 3 months. Viusid ${ }^{\circledR}$ was provided by Proximo Health Solucion, (distributor of Catalysis Laboratory, Spain in Bangladesh).

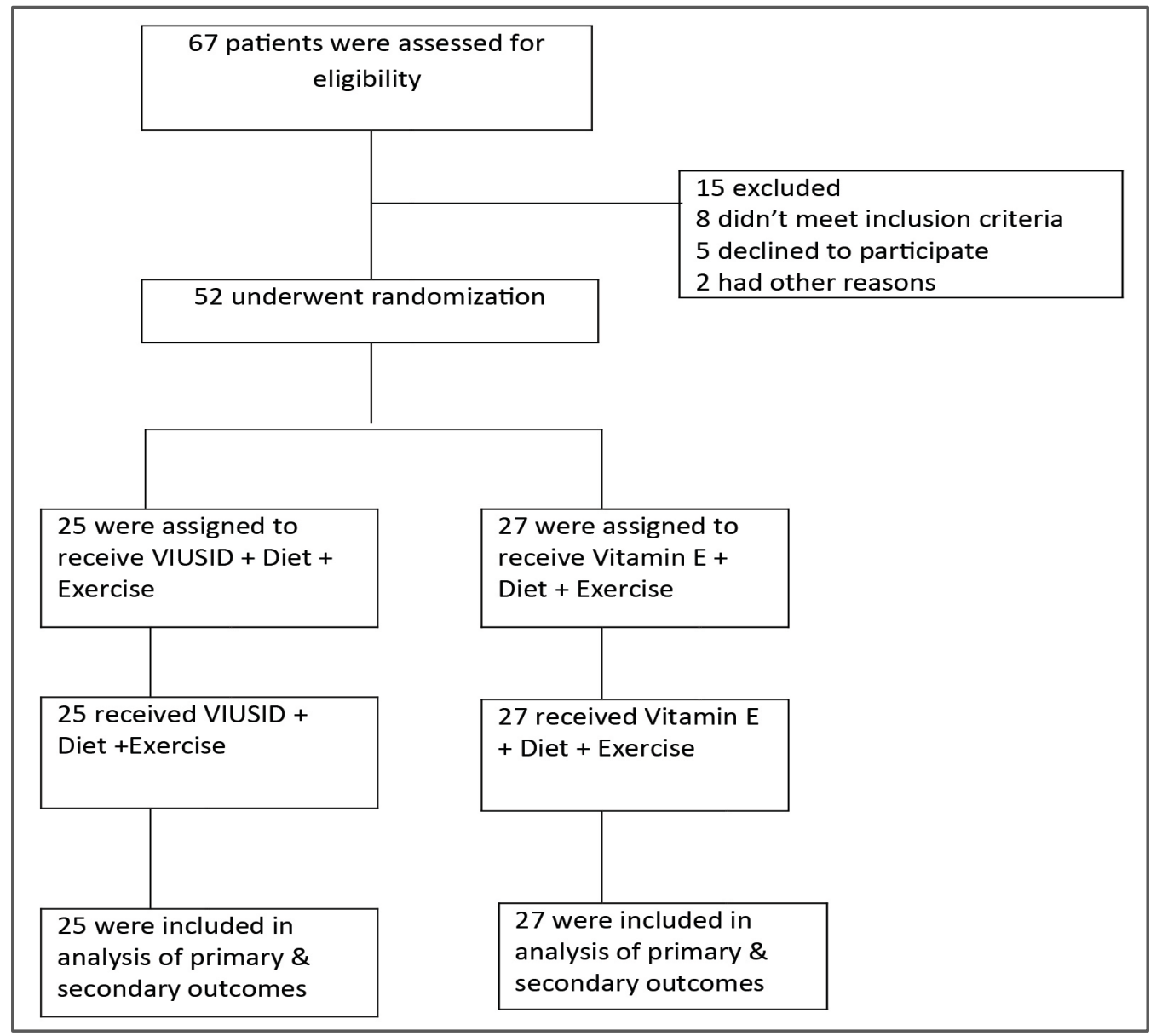

Figure 2. The trial profile of participants who completed follow up 
Table 2. The baseline characteristics of patients

\begin{tabular}{|l|c|c|}
\hline Variable & Viusid $^{\circledR}$ group (n=25) & Vitamin E group (n=27) \\
\hline Weight $(\mathrm{kg})$ & $80.48 \pm 4.24$ & $79.15 \pm 4.54$ \\
\hline BMI $\left(\mathrm{kg} / \mathrm{m}^{2}\right)$ & $29.24 \pm 2.57$ & $29.15 \pm 2.96$ \\
\hline ALT $(\mathrm{U} / \mathrm{L})$ & $114 \pm 25.9$ & $105 \pm 15.5$ \\
\hline AST $(\mathrm{U} / \mathrm{L})$ & $78.4 \pm 12.28$ & $73.26 \pm 9.94$ \\
\hline Triglyceride $(\mathrm{mg} / \mathrm{dL})$ & $230.52 \pm 23.59$ & $219.33 \pm 23.2$ \\
\hline Cholesterol $(\mathrm{mg} / \mathrm{dL})$ & $196.72 \pm 19.10$ & $190.29 \pm 15.39$ \\
\hline Steatosis score $(\mathrm{dB} / \mathrm{m})$ & $286 \pm 16.3$ & $278 \pm 14.4$ \\
\hline Fibrosis score $(\mathrm{kPa})$ & $6.8 \pm 0.5$ & $6.9 \pm 0.5$ \\
\hline
\end{tabular}

AST: Aspartate aminotransferase. ALT: Alanine aminotransferase

Clinical assessment, including body weight, BMI, liver function tests (ALT \& AST) and lipid profile along with compliance with the study medication (verified through sachet count) and adverse events, were determined at baseline and / or at monthly intervals during the 3 months of the study. Improvement of histological features of NASH (steatosis \& fibrosis score) was assessed using Fibroscan in both groups before starting the treatment (baseline) and after 3 months of treatment. The standard fibroscan scoring system to evaluate the changes in histologic features like liver stiffness (fibrosis) and steatosis is defined \& shown in Table 3. Fibroscan (Echosensfibroscan, probe FF01381) was done in Lab-Aid Diagnostic Centre in Dhaka, under the supervision of a single hepatologist who was unaware of the patients clinical and biochemical data, treatment assignment.

\section{Primary endpoints (histological):}

- Improvement in steatosis score [mean CAP value $(\mathrm{dB} / \mathrm{m})]$

- Improvement in fibrosis score [mean E value $(\mathrm{kPa})]$

\section{Secondary endpoint (biochemical):}

- Improvement in ALT [(mean value (IU/L)] levels

Statistical analysis: The primary analysis was an intention-to-treat analysis in which the proportions of subjects in Viusid $^{\mathbb{B}}$ group in whom there was improvement in the histological scores (steatosis and fibrosis) of nonalcoholic steatohepatitis were compared with the proportion of subjects in the Vitamin E group in whom there was improvement. The results were expressed as means \pm standard deviation. Comparisons were made with the use of the Welch Two Sample t-test. Within each group, post-treatment weight, lipid profile, ALT \& AST were compared using Wilcoxon's signed rank test. For all comparisons, $\mathrm{P}<0.05$ was considered to be statistically significant.

Table 3A. Steatosis grading reference in CAP value $(\mathrm{dB} / \mathrm{m})$

\begin{tabular}{|c|c|c|}
\hline CAP Value (dB/m) & Steatosis Stage & Status \\
\hline$<215$ & S0 & $<10 \%$ of hepatocytes \\
\hline $216-252$ & S1 & $11-33 \%$ of hepatocytes \\
\hline $252-296$ & S2 & $34-66 \%$ of hepatocytes \\
\hline$>296$ & S3 & $67 \%$ of hepatocytes \\
\hline
\end{tabular}

Table 3B. Fibrosis grading reference in $\mathrm{E}$ value $(\mathrm{kPa})$

\begin{tabular}{|c|c|l|}
\hline E Value (kPa) & Fibrosis Stage & \multicolumn{1}{|c|}{ Status } \\
\hline $0-5.9$ & F0 & No fibrosis \\
\hline $5.9-7.1$ & F1 & Portal fibrosis without septa \\
\hline $7.1-8.1$ & F2 & Portal fibrosis with few septa \\
\hline $8.8-10.2$ & F3 & Numerous septa without cirrhosis \\
\hline $11.7-75$ & F4 & Cirrhosis \\
\hline
\end{tabular}

\section{Results}

52 subjects $($ Males $=34 \&$ Females $=18$; Mean age $=$ $46.2 \pm 8.5$ years), who met the inclusion and exclusion criteria, were included in the analysis. No patient had clinical evidence of diabetes, cirrhosis or decompensated liver disease. There was no patient with a BMI greater than 35 in our study. At baseline, there was no significant difference between two groups with respect to mean age, BMI, lipid profile, steatosis, fibrosis, ALT \& AST levels. The baseline characteristics of these patients are shown in Table 2. At the end of study, the weight reduction in both groups was similar. The mean weight reduction was from $80.48 \pm 4.24 \mathrm{~kg}$ to $75.32 \pm 4.64 \mathrm{~kg}$ in Viusid $^{\circledR}$ group vs. from $79.15 \pm 4.54 \mathrm{~kg}$ to $74.59 \pm 4.55 \mathrm{~kg}$ in Vitamin E group $(\mathrm{p}=0.438)$. After 3 months of treatment, significant improvements were seen in both endpoints from baseline data in Viusid ${ }^{\mathbb{B}}$ group compared to Vitamin E group (Figure 3). Treatment with Viusid ${ }^{\circledR}$, as compared with Vitamin E, was associated with a significant reduction of both mean steatosis score [from S2-S3 (CAP: 286 \pm 16.3 $\mathrm{dB} / \mathrm{m}$ ) to $\mathrm{S} 0-\mathrm{S} 1$ (CAP: $208 \pm 18.5 \mathrm{~dB} / \mathrm{m}$ ) in Viusid $^{\circledR}$ group vs. from S2-S3 (CAP: $278 \pm 14.4 \mathrm{~dB} / \mathrm{m}$ ) to $\mathrm{S} 1-\mathrm{S} 2$ (CAP: $253 \pm 12.1 \mathrm{~dB} / \mathrm{m})$ in Vitamin $E$ group $(\mathrm{p}<0.00001)]$ and fibrosis score [from F1-F2(E: $6.8 \pm 0.5 \mathrm{kPa}$ ) to F0-F1 (E: $5.1 \pm 0.7 \mathrm{kPa}$ ) in Viusid ${ }^{\circledR}$ group vs. from F1-F2 (E: $6.9 \pm 0.5$ $\mathrm{kPa})$ to $\mathrm{F} 1-\mathrm{F} 2(\mathrm{E}: 6.5 \pm 0.4 \mathrm{kPa})$ in Vitamin $\mathrm{E}$ group $(p<0.00001)]$. Similarly, the mean alanine transaminase (ALT) levels significantly decreased from $114 \pm 25.9 \mathrm{U} / \mathrm{L}$ to $43 \pm 9.1 \mathrm{U} / \mathrm{L}$ in Viusid ${ }^{\circledR}$ group compared to from $105 \pm 15.5$ $\mathrm{U} / \mathrm{L}$ to $55 \pm 11.7 \mathrm{U} / \mathrm{L}$ in Vitamin $\mathrm{E}$ group ( $>0.00001$ ). The mean Aspartate aminotransferase (AST) levels also decreased from $78.4 \pm 12.28 \mathrm{U} / \mathrm{L}$ to $47.36 \pm 7.39 \mathrm{U} / \mathrm{L}$ in Viusid $^{\circledR}$ group vs. from $73.26 \pm 9.94 \mathrm{U} / \mathrm{L}$ to $55.29 \pm 11.26$ $\mathrm{U} / \mathrm{L}$ in Vitamin $\mathrm{E}$ group $(\mathrm{p}<0.00001)$. 
Table 4. The comparison between baseline and after 3 months data of both groups

\begin{tabular}{|l|c|c|c|c|c|}
\hline \multirow{2}{*}{ Variables } & \multicolumn{2}{c|}{ Viusid Group } & \multicolumn{2}{c|}{ Vitamin E Group } & \multirow{2}{*}{ P value } \\
\cline { 2 - 5 } & Basal & After 3 months & Basal & After 3 months & \\
\hline Weight $(\mathrm{kg})$ & $80.84 \pm 4.24$ & $75.32 \pm 4.64$ & $79.15 \pm 4.54$ & $74.59 \pm 4.55$ & 0.438 \\
\hline BMI $\left(\mathrm{kg} / \mathrm{m}^{2}\right)$ & $29.24 \pm 2.57$ & $28.16 \pm 2.3$ & $29.15 \pm 2.96$ & $28.32 \pm 2.84$ & 0.7876 \\
\hline ALT $(\mathrm{U} / \mathrm{L})$ & $114 \pm 25.9$ & $43 \pm 9.1$ & $105 \pm 15.5$ & $55 \pm 11.7$ & $>0.00001$ \\
\hline AST $(\mathrm{U} / \mathrm{L})$ & $78.4 \pm 12.28$ & $47.36 \pm 7.39$ & $73.26 \pm 9.94$ & $55.29 \pm 11.26$ & $>0.00001$ \\
\hline Triglyceride $(\mathrm{mg} / \mathrm{dl})$ & $230.52 \pm 23.59$ & $168.68 \pm 18.13$ & $219.33 \pm 23.2$ & $180.41 \pm 19.75$ & $>0.0001$ \\
\hline Cholesterol $(\mathrm{mg} / \mathrm{dl})$ & $196.72 \pm 19.10$ & $177.88 \pm 15.36$ & $190.29 \pm 15.39$ & $174.4 \pm 18.92$ & 0.4786 \\
\hline Steatosis score $(\mathrm{dB} / \mathrm{m})$ & $286 \pm 16.3$ & $208 \pm 18.5$ & $278 \pm 14.4$ & $253 \pm 12.1$ & $<0.00001$ \\
\hline Fibrosis score $(\mathrm{kPa})$ & $6.8 \pm 0.5$ & $5.1 \pm 0.7$ & $6.9 \pm 0.5$ & $6.5 \pm 0.4$ & $<0.00001$ \\
\hline
\end{tabular}

There was significant reduction of triglyceride level in both Vitamin E and Viusid ${ }^{\circledR}$ group; however, the reduction was statistically significant in Viusid $^{\circledR}$ group (mean triglyceride level reduces from $230.52 \pm 23.59$ to $168.68 \pm 18.13 \mathrm{mg} / \mathrm{dL}$ in Viusid $^{\circledR}$ group vs. from $219.33 \pm 23.2$ to $180.41 \pm 19.75 \mathrm{mg} / \mathrm{dL}$ in Vitamin E group; $\mathrm{p}>0.0001$ ). Like Vitamin E, there was a significant correlation between the normalization of triglyceride levels and the improvement of steatosis in the patients assigned to the Viusid $^{\circledR}$ group. However, these are only preliminary observations that should be interpreted carefully and investigated in further studies to compare the results. The comparison between baseline and data of both groups after 3 months are shown in Table 4.

No patient was diagnosed as diabetic during the trial. Mild GI disturbances like nausea, dyspepsia and diarrhea were reported in two subjects who received Viusid ${ }^{\circledR}$. However, there was no incidence of discontinuation or dose modification of Viusid ${ }^{\circledR}$ secondary to these adverse events.

\section{Discussion}

Nonalcoholic steatohepatitis (NASH) with progressive fibrosis has become a global challenge as advanced liver fibrosis results in cirrhosis, liver failure and often requires liver transplantation. The prevalence of NAFLD may be increasing because of changing of dietary habits, less physical activity, and increasing incidence of obesity. In most patients oxidative stress is thought to be the cause of lipid accumulation in the hepatocytes and hepatic inflammation and fibrosis[16]. There is no strong evidence supporting any effective therapeutic agents for reducing inflammation and fibrosis or preventing the progression of NAFLD. Nonetheless, weight reduction and the drugs that overcome oxidative stress and inflammatory markers may be appropriate to slow the disease process $[16,17]$. The aim of our study was to compare the efficacy of two potential anti-oxidant agents (Vitamin E and Viusid ${ }^{\mathbb{B}}$ ) when combined with lifestyle modification in improving histological \& biochemical parameters in fibrotic NASH patients.

Vitamin E ( $\alpha$-tocopherol), a type of lipid-soluble, chain-breaking antioxidant, is an essential micronutrient that can protect cellular structural integrity against damage from lipid peroxidation and oxygen-free radicals [17]. In vivo and in vitro studies have demonstrated that vitamin $\mathrm{E}$ attenuated NASH via multiple mechanisms, including up-regulation of superoxide dismutase activity and inhibition of genes related to inflammation, fibrosis and hepatocellular necrosis $[17,18]$. A recent meta-analysis demonstrated that vitamin E supplementation had a significant and positive effect in the improvement of steatosis, ballooning degeneration, lobular inflammation and fibrosis in patients with NASH [17]. In addition, Vitamin $E$ at daily doses of $800 \mathrm{IU} /$ day is recommended as a first line pharmacotherapy for non-diabetic adults with biopsy- proven NASH by the American Association for the Study of Liver Diseases (AASLD) [7].

On the other hand, Viusid ${ }^{\circledR}$ is an effective hepatoprotector with recognized antioxidant, immunomodulatory and anti-fibrotic properties that contains different antioxidant micro-nutrients like Glycyrrhizic acid, Glucosamine, Ascorbic acid, Zinc and others (Table 1).

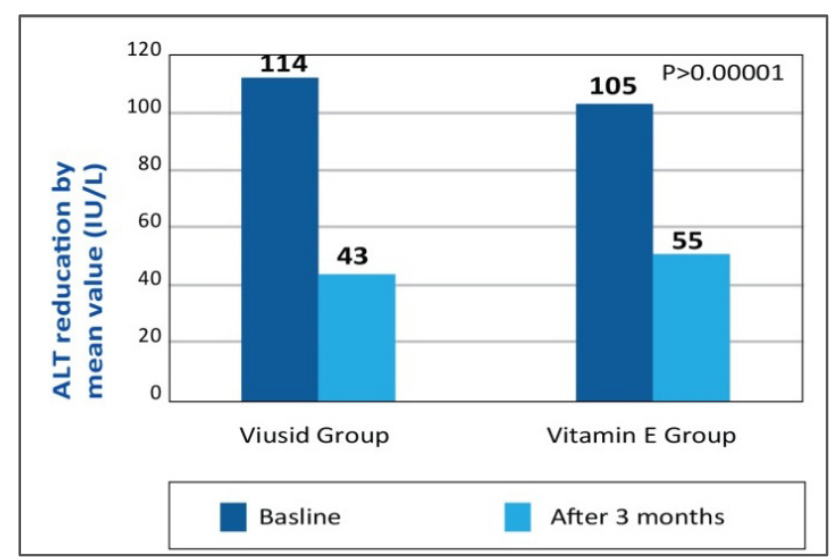

Figure 3A. Mean ALT values at baseline and after 3 months of treatment in both the groups 


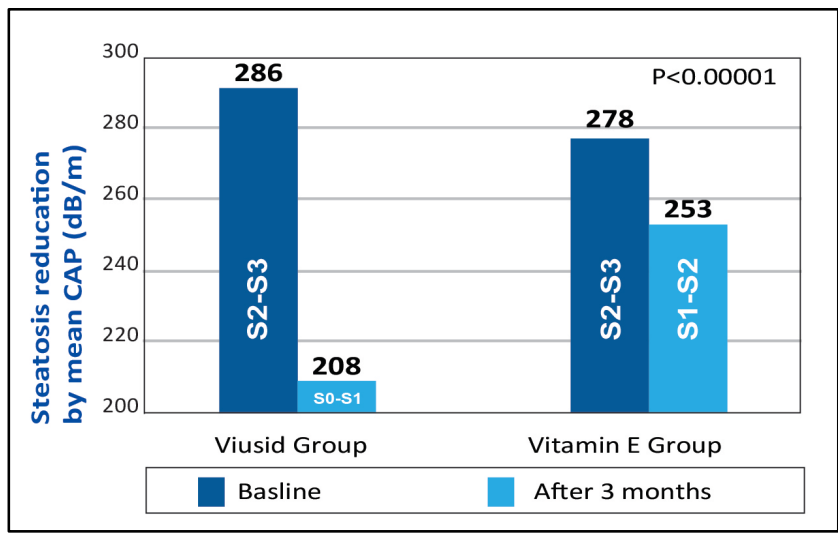

Figure 3B. Mean steatosis scores at baseline and after 3 months of treatment in both the groups

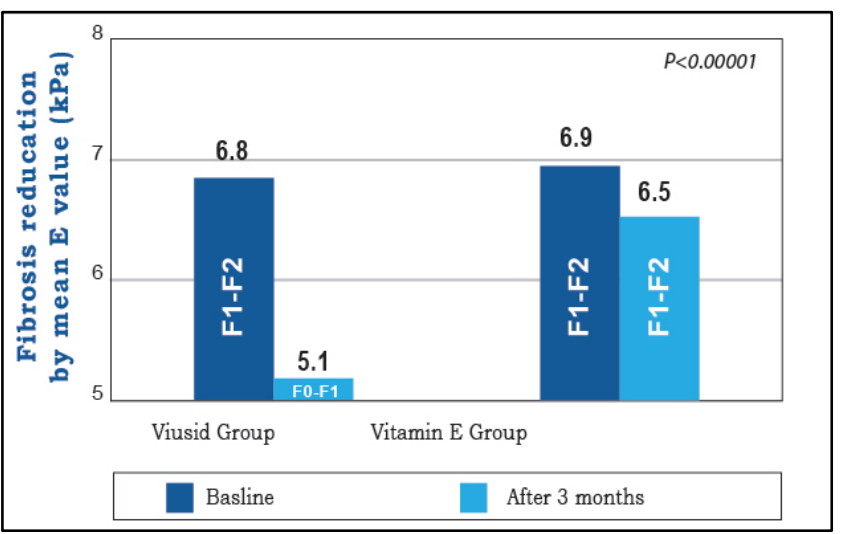

Figure 3C. Mean fibrosis scores at baseline and after 3 months of treatment in both the groups

Viusid $^{\circledR}$ is a powerful antioxidant because of the Antioxidant activity of glycyrrhizic acid is over 100 times stronger than that of Vitamin $\mathrm{E}$ and the antioxidant capacity of Viusid ${ }^{\circledR}$ is further increased by "Molecular Activation", which is about $800 \%$ more potent than its previous non-activated state $[9,10,11,12]$. Evidences showed that Viusid $^{\circledR}$ inhibits oxidative stress, lipid per-oxidation (MDA) and several pro-inflammatory cytokines (TNF-alpha, IL-1 \&IL-6) involved in fibrogenesis process. Moreover it demonstrated an anti-inflammatory effect (increased production of IL-10), anti-apoptotic effect of healthy hepatocyte, apoptotic stimulating effect of hepatic stellate cells (HSC), hepatocyte proliferating effect and stabilizing effect of hepatic cellular membranes which are potential for "Anti-fibrotic Activity"[13, 14]. Encouraging effects of Viusid $^{\circledR}$ on liver histology and biochemistry have been reported in patients with non-alcoholic fatty liver disease and chronic hepatitis $\mathrm{C}$. The authors reported that the addition of Viusid $^{\circledR}$ to the conventional interferon/ribavirin therapy was associated with significant histological and biochemical improvements, especially in patients without a sustained virological response [15]. In another study the same authors showed that the administration of Viusid ${ }^{\mathbb{B}}$ combined with a lifestyle modification based on a hypocaloric diet and exercise during 6 months was associated with marked histological improvements in steatosis, lobular inflammation, ballooning and NAFLD activity score in patients with non-alcoholic fatty liver disease [8]. Additionally, another study demonstrated a significant biochemical improvement of Viusid $^{\mathbb{B}}$ in achieving ALT normalization in NAFLD patients [9].

This has led to enthusiasm over the possibility that Viusid $^{\circledR}$ may have more benefits than Vitamin $E$ in improving histological \& biochemical parameters in fibrotic NASH patients. The present study has shown that in fibrotic NASH patients, Viusid ${ }^{\mathbb{B}}$ was superior to Vitamin $\mathrm{E}$ in improving both histological parameters (mean steatosis and mean fibrosis score) on fibroscan and biochemical parameters (ALT, AST). Both the drugs were well tolerated and only mild GI disturbances were reported in Viusid ${ }^{\circledR}$ group. In the current study, we found that serum triglyceride levels were modified in both groups either taking Vitamin $\mathrm{E}$ or Viusid $^{\mathbb{B}}$; however, they were significantly reduced in the patients treated with Viusid ${ }^{\mathbb{B}}$. There is no evidence available to support the molecular basis of this mechanism; however, some component of Viusid $^{\circledR}$, such as folic acid, could be linked to the improvement of methylation reactions that play pivotal roles in the function of hepatocytes, including the prevention of triglyceride accumulation in the liver, the production of the antioxidant glutathione and the preservation of cell membrane integrity [15].

The main strength of this study was determined by the excellent patients' compliance with both treatments. A weakness of our study was that patients were treated for 3 months only and it remains unclear whether the biochemical and histological improvements can be sustained beyond 3 months. Continued dietary intervention for long periods could lead loss of treatment adherence, which might eventually reverse any beneficial effect on hepatic histology. For these reasons, long-term studies that support the continued efficacy of dietary intervention are needed.

\section{Conclusions}

Viusid $^{\circledR}$ was superior to Vitamin $\mathrm{E}$ in reducing steatosis\& fibrosis score in Nondiabetic and Noncirrhotic NASH patients. Further randomized, controlled and large scale comparative clinical trial is needed to better assess the value of Viusid ${ }^{\circledR}$ for fibrotic NASH management.

\section{Acknowledgements}

Declaration of personal interests: We are thankful to the study participants for giving consent to perform this study. We gratefully acknowledge the technical and supporting staff of Hepatology Department. The authors were collectively responsible for the study design, data statistical analysis and interpretation of data; the writing of the 
manuscript; and the decision to submit the manuscript for publication. This was an investigator-initiated proposal and Proximo Health Solucion (distributor of Catalysis Laboratory, Spain in Bangladesh) had no direct involvement in the design of the study, data collection, or preparation of the manuscript. The lead author wrote the first draft of the manuscript, and subsequent drafts were reviewed by all authors. No writing support was received.

Declaration of funding interests: Supported in part by a grant from Proximo Health Solucion. They provided the Viusid $^{\circledR}$ sachets for the study protocol. The supporting sources had no role in the study design, collection of data, interpretation of data, writing of the manuscript or submission for publication.

\section{REFERENCES}

[1] Chalasani N, Younossi Z, Lavine J, et al. AASLD practice guideline. The diagnosis and management of non-alcoholic fatty liver disease: practice Guideline by the American Association for the Study of Liver Diseases, American College of Gastroenterology, and the American Gastroenterological Association. Hepatology 2012; 5:2005-23.

[2] Watanabe S, Hashimoto E, Ikejima K, et al. Evidence-based clinical practice guidelines for nonalcoholic fatty liver disease/nonalcoholic steatohepatitis. Hepatol Res 2015; 45:363-77.

[3] Ahmed A, Wong RJ, Harrison SA. Nonalcoholic fatty liver disease review: diagnosis, treatment, and outcomes. Clin Gastroenterol Hepatol 2015; 13:2062-70.

[4] Shahinul Alam, Golam Azam, et al. Past, Present, and Future of Hepatitis B and Fatty Liver in Bangladesh.GastroenterolHepatol Open Access 2017, 6(3): 00197

[5] DetlefSchuppan, M Schattenberg, et al. Non-alcoholic steatohepatitis: Pathogenesis and novel therapeutic approaches. Journal of Gastroenterology and Hepatology2013; 28 (Suppl. 1): 68-76

[6] Himanshu Rana, Suraj Singh Yadav, et al. Comparative Effect of Insulin Sensitizers and Statin on Metabolic Profile and Ultrasonographical Score in Non Alcoholic Fatty Liver Disease. Journal of Clinical and Diagnostic Research. 2016 Aug, Vol-10(8): OC19-OC23

[7] Pathik Parikh, Meghraj Ingle, et al.An open-label randomized control study to compare the efficacy of Vitamin E versus ursodeoxycholic acid in nondiabetic and noncirrhotic Indian NAFLD patients. Saudi J Gastroenterol 2016; 22:192-7.

[8] Eduardo Vilar Gomez et al., Viusid, a nutritional supplement, in combination with interferon $\mathrm{a}-2 \mathrm{~b}$ and ribavirin in patients with chronic hepatitis C. Liver International ISSN 1478-3223.

[9] Mahtab MA et al.: Viusid, a nutritional supplement, in combination with diet and exercise in treating patients with non-alcoholic steatohepatitis. Bangladesh Liver Journal 2009; 1(2): p 9-11.

[10] Monica Damle et al.: Glycyrrhiza glabra(Liquorice) - a potent medicinal herb. International Journal of Herbal Medicine 2014; 2(2): 132-136.

[11] Gonzalez A., Antioxidants: Study \& Applications, Internal Editions of Catalysis Laboratories, 2005.

[12] Mercedes Montero et al., Effects of Kalsis, A Dietary Supplement, on Bone Metabolism in the Ovariectomized Rats. Journal of Osteoporosis, Volume 2012, Article ID 639427, 7 pages; doi:10.1155/2012/639427.

[13] Eduardo Vilar Gomez et al., Viusid, a nutritional supplement, increases survival and reduces disease progression in HCV-related decompensated cirrhosis: a randomized and controlled trial. BMJ Open. 2011; 2:e000140. Doi: 10.1136/bmjopen-2011-000140.

[14] Eduardo Vilar Gomez et al., Antioxidant and immunomodulatory effects of Viusid in patients with chronic hepatitis C. World J Gastroenterol 2010 June 7; 16(21): 2638-2647.

[15] E. Vilar Gomez et al., A nutritional supplement Viusid, in combination with diet and exercise, in patients with nonalcoholic fatty liver disease. Aliment Pharmacol Ther 30, 999-1009.

[16] Hajiani E, et al. Comparison of therapeutic effects of silymarin and vitamin e in nonalcoholic fatty liver disease: Results of an open-labele, prospective, randomized study. Jundishapur Journal of Natural Pharmaceutical Products 2009; 4(1): 8-14.

[17] RenfanXu, Anyu Tao, et al. Association between vitamin E and non-alcoholic steatohepatitis: a meta-analysis. Int J Clin Exp Med 2015; 8(3):3924-3934.

[18] Phung N, Pera N, Farrell G, Leclercq I, Hou JY, George J. Pro-oxidant-mediated hepatic fibrosis and effects of antioxidant intervention in murine dietary steatohepatitis. Int J Mol Med 2009; 24: 171-80. 